\title{
Aberrant Structural Brain Connectivity in Adolescents with Attentional Problems Who Were Born Prematurely
}

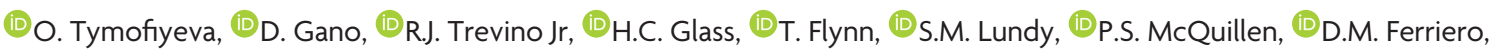 \\ (1)A.J. Barkovich, and ${ }^{D} \mathrm{D}$. Xu
}

\begin{abstract}
BACKGROUND AND PURPOSE: Differences in structural brain connectivity that underlie inattention have been previously investigated in adolescents with attention deficit/hyperactivity disorder, but not in the context of premature birth, which is often associated with attentional problems. The purpose of this study was to identify the neural correlates of attentional problems in adolescents born prematurely and determine neonatal predictors of those neural correlates and attention problems.
\end{abstract}

MATERIALS AND METHODS: The study included 24 adolescents (12.5 \pm 1.8 years of age; 12 girls, 12 boys) who were born prematurely and underwent MR imaging of the brain and cognitive assessment, both shortly after birth and as adolescents. Structural connectivity was assessed at adolescence using diffusion tensor imaging and tractography.

RESULTS: Of the 24 subjects, 12 had attention deficits. A set of axonal pathways connecting the frontal, parietal, temporal, and occipital lobes had significantly lower fractional anisotropy in subjects with attentional problems. The temporoparietal connection between the left precuneus and left middle temporal gyrus was the most significantly underconnected interlobar axonal pathway. Low birth weight and ventriculomegaly, but not white matter injury or intraventricular hemorrhage on neonatal MR imaging, predicted temporoparietal hypoconnectivity in adolescence. However, neither birth weight nor other neonatal characteristics were associated with attention deficits directly.

CONCLUSIONS: We identified an aberrant structural brain connectivity pattern, involving temporoparietal hypoconnectivity, in prematurely born adolescents with attentional problems. We also identified birth weight as a potential neonatal predictor of the temporoparietal hypoconnectivity. These findings add to our understanding of the neural basis and etiology of inattention in adolescents after premature birth.

ABBREVIATIONS: $A D H D=$ attention deficit/hyperactivity disorder; $A A L=$ Automated Anatomical Labeling; FA = fractional anisotropy; FDR $=$ false discovery rate; TOVA = Test of Variables of Attention; $\mathrm{WMI}=$ white matter injury

A bout 1 in 10 children are born prematurely and, as a result, enter the world with a higher risk of several health problems as well as early death. ${ }^{1}$ Those who survive often have life-long

Received May 11, 2018; accepted after revision August 16

From the Departments of Radiology \& Biomedical Imaging (O.T., R.J.T., T.F., A.J.B., D.X.), Neurology (D.G., H.C.G., D.M.F.), and Pediatrics (D.G., H.C.G., S.M.L., P.S.M., D.M.F.), University of California, San Francisco, San Francisco. California.

This study was supported by P01NS082330 to D.M.F., P.S.M., D.X., and A.J.B.; NICHD R01HD072074 to D.X. and O.T.; NCCIH R21AT009173 to O.T.; and the University of California, San Francisco, Research Evaluation and Allocation Committee and the J. Jacobson Fund to O.T. and D.X.

The content of this article is solely the responsibility of the authors and does not necessarily represent the official views of the National Institutes of Health or other funding agencies. The funding agencies did not play any role in study design or in the collection, analysis, and interpretation of data; in the writing of the report; and in the decision to submit the article for publication.

Please address correspondence to Olga Tymofiyeva, PhD, Department of Radiology \& Biomedical Imaging, University of California, San Francisco, 1700 4th St, Byers Hall, Suite 102, San Francisco, CA 94158; e-mail: Olga.Tymofiyeva@ucsf.edu

- Indicates open access to non-subscribers at www.ajnr.org

http://dx.doi.org/10.3174/ajnr.A5834 neurodevelopmental problems, with attention deficit being among the most consistently reported cognitive impairment related to prematurity. ${ }^{2}$ This deficit impacts daily functioning, and many individuals meet the formal diagnostic criteria for attention deficit/hyperactivity disorder (ADHD). ${ }^{3}$ It is essential to better understand the underlying neurologic causes of attentional problems and their etiologies to develop effective interventions.

Diffusion tensor imaging allows noninvasive assessment of white matter microstructure. ${ }^{4}$ This methodology enables $3 \mathrm{D}$ visualization of white matter properties and has been used for several decades to study neurologic and psychiatric disorders in an attempt to relate white matter abnormalities to clinical manifestations. ${ }^{5}$ A commonly used metric derived from DTI is fractional anisotropy (FA). A general, simplified interpretation is that higher FA represents higher white matter integrity in specific brain regions. With regard to attentional problems, several studies have focused on the FA in the brains of individuals with ADHD, reporting both higher ${ }^{6-8}$ and lower ${ }^{9-11}$ FA values compared with healthy controls. The effects of premature birth on 
DTI parameters have also been investigated, showing that children born prematurely tend to have lower FA values in several regions of the brain consistently from infancy to adulthood. ${ }^{12-16}$

Recently, neurologic and psychiatric disorders, including ADHD, are increasingly being viewed not as anomalies of focal brain regions but rather as disorders arising from disruption of distributed brain networks (connectomes). ${ }^{17}$ These networks are modeled as sets of nodes, with pairs of nodes connected by edges. In a structural connectivity study, cortical and subcortical gray matter structures typically play the role of nodes, whereas tractography-derived white matter connections between them serve as edges. This network approach differs in an important way from the voxel-based DTI approaches in that it moves away from focal abnormalities to network-level disruptions and offers new explanations of observed dysfunctions.

To date, there are 3 studies examining white matter network properties in youth with ADHD. ${ }^{18-20}$ The main findings include aberrant connectivity of the frontal and striatal regions, ${ }^{18-20}$ but also parietal, ${ }^{18,20}$ occipital, ${ }^{18,20}$ temporal, ${ }^{20}$ and cerebellar regions. ${ }^{19}$ One additional study used T1-weighted images and showed differences in topologic properties of structural covariance networks in youth with ADHD compared with controls. ${ }^{21}$ However, there have been no studies examining structural connectivity associated with inattention in adolescents born prematurely. This is an important gap in knowledge because understanding the underlying mechanisms of attentional dysfunction is crucial for the development of targeted interventions. The purpose of this study was to identify network-level neural correlates of attentional problems in adolescents born prematurely and assess neonatal predictors of those neural correlates.

\section{MATERIALS AND METHODS \\ Participants and Clinical Information}

The study was approved by the local institutional review board, and all participants in the study provided written informed assent, and their parents or legal guardians provided written informed consent in accordance with the Declaration of Helsinki. Prematurely born adolescents who were enrolled in a longitudinal MR imaging study of preterm infants were invited to return for optional MR imaging and neuropsychological testing at 10-14 years of age. Inclusion criteria for the cohort were birth before 33 weeks' gestation. Exclusion criteria included clinical evidence of a congenital malformation or syndrome, congenital infection, or clinical status too unstable for MR imaging. The group included in this study consisted of 24 adolescents (10-14 years of age; average age, $12.5 \pm 1.8$ years; 12 girls and 12 boys) who were born prematurely (including 11 extremely premature subjects, $<28$ weeks' gestation) and came for the 10- to 14-year follow-up visit and had both neuropsychological and diffusion MR imaging data available.

\section{Neonatal Clinical Assessments}

Trained research nurses blinded to the MR imaging findings reviewed medical records and extracted clinical data. Maternal and antenatal variables included exposure to prenatal steroids as well as maternal age, primiparity, maternal smoking, placenta previa, preeclampsia, and twin gestation.
Demographic variables included gestational age at birth, birth weight, and sex. The $z$ score for birth weight was calculated. Perinatal variables included placental abruption, chorioamnionitis, and mode of delivery, which was classified as vaginal or cesarean delivery. Chorioamnionitis was diagnosed clinically (maternal fever, $>38^{\circ} \mathrm{C}$ during labor or fetal tachycardia with uterine tenderness, treated with antibiotics). ${ }^{22}$ Neonatal variables included duration of mechanical ventilation, infection, hypotension, symptomatic patent ductus arteriosus, necrotizing enterocolitis, neonatal surgery, and chronic lung disease. Neonates with culture-positive sepsis, clinical signs of sepsis with blood culture negative for sepsis, or meningitis were classified as having infection. Hypotension was defined as a period of sustained low blood pressure treated with intravenous fluid bolus and/or inotropes. Neonates with clinical signs of patent ductus arteriosus (prolonged systolic murmur, bounding pulses, and hyperdynamic precordium) and evidence of left-to-right flow through the patent ductus arteriosus on echocardiogram were classified as having symptomatic patent ductus arteriosus. Necrotizing enterocolitis was diagnosed according to the Bell stage 2 criteria. ${ }^{23}$ Chronic lung disease was defined as an oxygen requirement at 36 weeks' postmenstrual age.

\section{Neonatal MR Imaging}

MR imaging scans were obtained after birth as soon as the neonates were clinically stable and again near term-equivalent age when possible. A custom MR imaging-compatible incubator with a specialized neonatal head coil was used to provide a quiet, well-monitored environment for the neonates, minimizing patient movement and improving the signal-to-noise ratio. ${ }^{24} \mathrm{MR}$ imaging scans were acquired using a $1.5 \mathrm{~T}$ scanner and a specialized, high-sensitivity, neonatal head coil built into the MR imaging-compatible incubator. MR imaging scans included axial spin-echo T2-weighted images $(\mathrm{TR}=3$ seconds; $\mathrm{TE}=60$ and 120 $\mathrm{ms}$; FOV $=240 \mathrm{~mm}$ with a $256 \times 256$ matrix; slice thickness $=4$ $\mathrm{mm}$; gap $=2 \mathrm{~mm}$ ) and sagittal volumetric $3 \mathrm{D}$ spoiled gradientecho $\mathrm{T} 1$-weighted images $(\mathrm{TR}=36 \mathrm{~ms}$; minimum $\mathrm{TE}$; FOV = $180 \mathrm{~mm} ; 1.0-\mathrm{mm}$ isotropic). A single pediatric neuroradiologist (A.J.B.) blinded to the clinical history (other than premature birth) evaluated all MR images. The severity of the white matter injury (WMI) on T1-weighted MR imaging was scored according to our published criteria as none, mild ( $\leq 3$ areas of signal abnormality, each $<2 \mathrm{~mm}$ in diameter), moderate ( $>3$ areas of signal abnormality or areas of signal abnormality of $>2 \mathrm{~mm}$ but $<5 \%$ of the hemisphere involved), or severe ( $>5 \%$ of hemisphere involved). ${ }^{25}$ WMI was further classified as absent/mild or moderate/severe. Neonates were diagnosed with mild ventriculomegaly if the largest atrial ventricular diameter (at the level of the glomus of the choroid plexus) measured $8-10 \mathrm{~mm}$ and with moderate/ severe ventriculomegaly if it measured $>10 \mathrm{~mm} .{ }^{26}$ Intraventricular hemorrhage was classified according to the Papile grading system. ${ }^{27}$

\section{Adolescent Attention Assessments}

A psychologist blinded to imaging findings administered performance-based measures and parent report forms targeting attention. Attentional problems in most subjects $(n=17$ of the 24 
Table 1: Neonatal clinical characteristics of the study participants ${ }^{a}$

\begin{tabular}{lccc}
\hline & \multicolumn{2}{c}{ Abnormal Attention } & \\
\cline { 2 - 3 } & No $(\boldsymbol{n}=12)$ & Yes $(\boldsymbol{n}=12)$ & P Value \\
\hline Male & 6 & 6 & 1.0 \\
Gestational age at birth (mean) (wk) & $29.4 \pm 1.83$ & $27.8 \pm 2.9$ & .093 \\
BW (median) (IQR) (g) & $1167(900-1365)$ & $875(708-898)$ & .043 \\
BW z score, (median) (IQR) & $-0.35(-1.05-0.48)$ & $-0.22(-0.73-0.34)$ & .82 \\
Cesarean delivery & 6 & 4 & .41 \\
Intubated at birth & 5 & 11 & .027 \\
Apgar 5 (median) (IQR) & $8(6.5-8)$ & $5.5(3.5-8)$ & .84 \\
Apgar 10 (median) (IQR) & $7(4-7)$ & $6.5(5-8)$ & .56 \\
Hypotension & 5 & 10 & .089 \\
Patent ductus arteriosus & 2 & 7 & .089 \\
Necrotizing enterocolitis & 0 & 5 & .037 \\
Chronic lung disease & 1 & 6 & .069 \\
Neonatal surgery & 2 & 8 & .036 \\
Infection & 2 & 10 & .022 \\
\hline Note-BW & & &
\end{tabular}

Note:-BW indicates birth weight; IQR, interquartile range.

${ }^{a}$ All neonates with necrotizing enterocolitis had surgery.

${ }^{\mathrm{b}}$ Kruskal-Wallis for continuous variables; Fisher exact test for categoric variables.

subjects included in this study) were evaluated using the Test of Variables of Attention (TOVA), a continuous performance test used to measure the speed and accuracy of attentional processing. ${ }^{28}$ Negative TOVA Attention Comparison Scores were interpreted as indicative of attentional problems. Six subjects were evaluated using the Conners Comprehensive Behavior Rating Scales, suitable for assessing children ages 6-18. Conners attention $t$-scores of $>60$ were interpreted as indicative of attentional problems. One subject was evaluated using the Child Behavior Checklist. $^{29}$ The Child Behavior Checklist attention $t$-scores of $>66$ were interpreted as indicative of attentional problems. The comparability of ADHD-related metrics has been previously reported for the TOVA and Child Behavior Checklist, ${ }^{30}$ for the Conners Rating Scales and Child Behavior Checklist, ${ }^{31,32}$ and for the TOVA and Conners Rating Scales. ${ }^{33}$

\section{Adolescent MR Imaging Data Acquisition and Processing}

Each adolescent underwent an hour-long MR imaging protocol using a 3T MR750 MR imaging scanner (GE Healthcare, Milwaukee, Wisconsin) and a 32-channel Nova Medical head coil (Siemens, Erlangen, Germany). The scan included a standard T1weighted sequence and a spin-echo echo-planar DTI sequence $(\mathrm{TR}=7.5$ seconds, minimum $\mathrm{TE}, \mathrm{FOV}=25.6 \mathrm{~cm}$ with a $128 \times$ 128 matrix, slice thickness $=2 \mathrm{~mm}$ ). Diffusion-sensitizing gradients were applied at a b-value of $1000 \mathrm{~s} / \mathrm{mm}^{2}$ along 30 noncollinear directions. An array spatial sensitivity encoding technique acceleration factor was set to 2 , resulting in a sequence scan time of 4 minutes.

Preprocessing was performed using the FMRIB Software Library (FSL 5.0.8; http://www.fmrib.ox.ac.uk/fsl) ${ }^{34}$ and Matlab (MathWorks, Natick, Massachusetts). A quality-assurance step was performed, in which diffusion volumes affected by motion were rejected ${ }^{35}$ and the remaining images were corrected for eddy current distortions, affine head motion, and b-vector rotation. DTI reconstruction and deterministic whole-brain streamline fiber tractography were performed using the Diffusion Toolkit (TrackVis; http://www.trackvis.org/dtk/). ${ }^{36}$ For whole-brain tractography, the Fiber Assignment by Continuous Tracking algorithm ${ }^{37}$ with 1 seed per voxel was applied using the entire dif- fusion-weighted volume as the mask image. Streamlines were terminated if the tract curvature exceeded $35^{\circ}$, a value chosen on the basis of previous work in adolescents. $^{38}$

Each brain was segmented into ROIs using the Automated Anatomical Labeling (AAL; https://omictools.com/aaltool) atlas. ${ }^{39}$ Only 90 cerebral regions were considered, because the cerebellum is often affected by stronger artifacts and is not always fully covered in the FOV. ${ }^{38}$ T1-weighted data were registered to the $\mathrm{B}_{0}$ volume of the DTI dataset and to the Montreal Neurological Institute space template using the FMRIB Linear Image Registration Tool (FLIRT; http://www. fmrib.ox.ac.uk/fsl/fslwiki/FLIRT). ${ }^{40,41}$ This step allowed the application of the AAL atlas in the DTI space to produce the 90 nodes of the network. The registration and segmentation results were visually inspected for errors. The resultant ROIs were dilated by 1 voxel. To define the connections between the ROIs, we considered only streamlines with at least 5 points, and we used the average FA value within voxels along streamlines connecting the ROIs as a proxy for the connectivity strength.

\section{Statistical Analysis}

Adolescent Structural Connectivity Analysis. Our main assumption was that aberrant connectivity underlies attentional problems. To test whether significant associations between connectivity and attention exists, we performed $t$ tests using attention as the grouping variable. We did not assume that the connections associated with this contrast of interest form a connected component. To control for the massive number of multiple comparisons, we applied a standard link-based controlling procedure, the false discovery rate (FDR). We used the implementation in Matlab by Zalesky et $\mathrm{al}^{42}$ with default parameters (a significance value of .05 and 1000 permutations). In this context, the FDR is referred to as a link-based controlling procedure because each link is treated independently for the sake of the family-wise error rate control.

The rest of the statistical analyses were performed using SPSS Statistics software (Version 25; IBM, Armonk, New York). Neonatal predictors of attentional problems in adolescence were tested using the Kruskal-Wallis test for continuous variables and the Fisher exact test for categoric variables. Neonatal predictors of abnormal structural connectivity associated with attentional problems in teens were investigated using a linear regression model with abnormal connectivity as the dependent variable and neonatal clinical and imaging metrics and the age at the adolescent MR imaging visit as independent variables. Additionally, the number of rejected diffusion volumes affected by motion was compared between the groups of adolescents with normal and abnormal attention using the independent-samples $t$ test.

No matching of intelligence quotient (IQ) was performed in the analyses because this can be problematic in the case of children 
Table 2: Neonatal scan results for study participants

\begin{tabular}{|c|c|c|c|}
\hline & \multicolumn{2}{|c|}{ Abnormal Attention } & \multirow[b]{2}{*}{$P$ Value } \\
\hline & No $(n=12)$ & Yes $(n=12)$ & \\
\hline Scan 1 & 12 & 12 & \\
\hline $\mathrm{WMI}^{\mathrm{a}}$ & & & .05 \\
\hline Absent & 6 & 8 & \\
\hline Mild & 1 & 3 & \\
\hline Moderate & 5 & 0 & \\
\hline Severe & 0 & 1 & \\
\hline $\mathrm{IVH}$ & & & .32 \\
\hline Absent & 8 & 11 & \\
\hline Grade I & 4 & 1 & \\
\hline Grade II & 0 & 0 & \\
\hline Grade III & 0 & 0 & \\
\hline Grade IV & 0 & 0 & \\
\hline VM & & & 1.0 \\
\hline Absent & 11 & 12 & \\
\hline Mild & 1 & 0 & \\
\hline Moderate/severe & 0 & 0 & \\
\hline Scan 2 & 10 & 8 & \\
\hline WMI & & & .57 \\
\hline Absent & 7 & 6 & \\
\hline Mild & 1 & 2 & \\
\hline Moderate & 2 & 0 & \\
\hline Severe & 0 & 0 & \\
\hline IVH & & & .35 \\
\hline Absent & 6 & 6 & \\
\hline Grade I & 4 & 1 & \\
\hline Grade II & 0 & 1 & \\
\hline Grade III & 0 & 0 & \\
\hline Grade IV & 0 & 0 & \\
\hline VM & & & .64 \\
\hline Absent & 9 & 8 & \\
\hline Mild & 0 & 0 & \\
\hline Moderate/severe & 1 & 0 & \\
\hline
\end{tabular}

Note:-IVH indicates intraventricular hemorrhage; VM, ventriculomegaly

${ }^{a}$ Note for WMI, scan 1, when dichotomized as absent/mild vs moderate/severe, $P=.16$. with attentional problems. On the traditional Wechsler IQ tests, the subtests tend to be rote, repetitive, and require attention and memory and are difficult for a child with attentional problems, and he or she may therefore perform slowly and/or inaccurately. ${ }^{43}$

\section{RESULTS}

Of the 24 adolescents, 12 have been determined to have attention deficits ( 6 girls/ 6 boys). There were no statistically significant differences in the number of rejected diffusion-weighted volumes, indicating that adolescents' movements during the MR imaging scan did not differ depending on the attentional problems $(t=$ $1.1, P=.28)$.

\section{Neonatal Predictors of Attentional Problems in Adolescence}

Details about the participants' neonatal characteristics grouped by attentional deficits can be found in Tables 1 and 2 . The groups did not differ significantly by sex, gestational age at birth, or birth weight $z$ scores (Table 1). However, there was a group difference in variables that denote increased illness severity, with infection, necrotizing enterocolitis, neonatal surgery, and intubation at birth reaching statistical significance (Table 1). Among neonatal scan results, only WMI at the first neonatal scan indicated a potential difference between the 2 groups with normal and abnormal attention $(P=.05)$ (Table 2$)$.

\section{Adolescent Structural Connectivity Correlates of Attentional Problems}

A set of tracts connecting frontal, parietal, temporal, and occipital lobes, including frontal cortices and subcortical regions, had significantly lower FA values in adolescents with attentional problems compared with those with normal attention. Connections
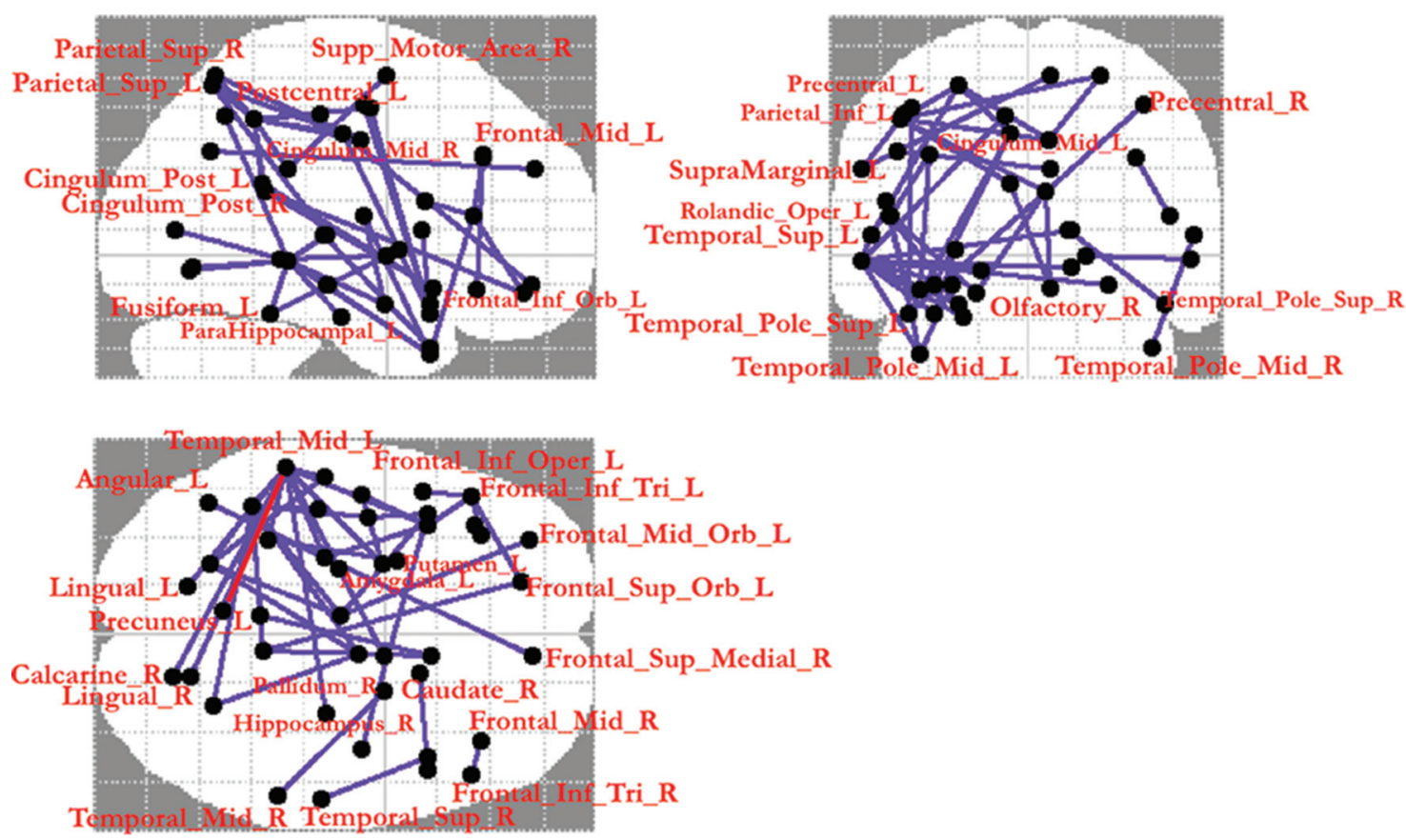

FIG 1. Results of the connection-wise group comparison of connectivity matrices using an FDR-corrected $t$ test with 1000 permutations $(P=$ .05). The connections highlighted in blue (46 edges, involving 44 nodes) were statistically significantly weaker in the attention deficit group. The connection highlighted in red, between the left precuneus and left middle temporal gyrus, was the most statistically significantly hypoconnected interlobular connection. 
Table 3: Structural connectivity analysis results (FDR-corrected) - connections with weaker connectivity in the inattention group that survived the FDR correction ${ }^{\mathrm{a}}$

\begin{tabular}{|c|c|c|}
\hline & Connection (AAL Abbreviation) & $\begin{array}{c}\text { Test } \\
\text { Statistic }\end{array}$ \\
\hline 1 & Frontal_Sup_Orb_L to Frontal_Inf_Tri_L & 3.39 \\
\hline 2 & Frontal_Mid_L to Frontal_Inf $\bar{T}$ ri_ $\overline{\mathrm{L}}$ & 4.12 \\
\hline 3 & Frontal_Inf_Ōper_L to Frontal_Inf_Tri_L & 4.16 \\
\hline 4 & Frontal_Mid_R to Frontal_Inf $\bar{T}$ ri $\bar{R}$ & 3.61 \\
\hline 5 & Frontal_Mid_L to Frontal_Inf_Orb__L & 3.75 \\
\hline 6 & Frontal_Mid_Orb_L to Cingulum_Mid_L & 2.82 \\
\hline 7 & Olfactory_R to Cingulum_Post_L ${ }^{-}$ & 4.56 \\
\hline 8 & Frontal_Sup_Orb_L to Cingulum_Post_R & 3.72 \\
\hline 9 & Olfactory $\mathrm{R}$ to Cingulum Post $\mathrm{R}$ & 4.01 \\
\hline 10 & Rolandic Oper L to Amygdala $\bar{L}$ & 2.74 \\
\hline 11 & Cingulum_Mid_L to Parietal_Sup_L & 4.12 \\
\hline 12 & Cingulum_Mid_R to Parietal_Sup_L & 3.87 \\
\hline 13 & Cingulum_Mid_R to Parietal_Sup_R & 3.52 \\
\hline 14 & Precentral_L to Parietal_Inf_L & 4.31 \\
\hline 15 & Cingulum_Mid_L to Parietal_Inf_L & 4.10 \\
\hline 16 & Cingulum_Mid_R to Parietal_Inf_L & 4.72 \\
\hline 17 & Cingulum Post $L$ to Parietal Inf $L$ & 4.11 \\
\hline 18 & Cingulum_Post_R to Parietal_Inf_L & 4.48 \\
\hline 19 & Postcentral_L to Parietal_Inf_L & 4.44 \\
\hline 20 & Parietal_Sup_L to Parietal_Inf_L & 5.40 \\
\hline 21 & Parietal Sup $R$ to Parietal $\operatorname{lnf} L$ & 3.45 \\
\hline 22 & Supp_Motor_Area_R to SupraMarginal_L & 2.34 \\
\hline 23 & Parietal_Inf_L to SupraMarginal_L & 3.48 \\
\hline 24 & Frontal_Sup_Medial_R to Angular_L & 2.6 \\
\hline 25 & Fusiform_L to Putamen_L & 3.49 \\
\hline 26 & Parietal_Sup_L to Temporal_Sup_L & 2.37 \\
\hline 27 & Precentral_L to Temporal_Pole_Sup_L & 3.73 \\
\hline 28 & Precentral_R to Temporal_Pole_Sup_L & 2.30 \\
\hline 29 & Cingulum_Mid_L to Temporal_Pole_Sup_L & 2.41 \\
\hline 30 & Caudate_R to Temporal_Pole_Sup_R & 3.33 \\
\hline 31 & Hippocampus_L to Temporal_Mid_L & 3.56 \\
\hline 32 & Hippocampus_R to Temporal_Mid_L & 3.75 \\
\hline 33 & ParaHippocampal_L to Temporal_Mid_L & 3.16 \\
\hline 34 & Amygdala $\mathrm{L}$ to Temporal Mid $\mathrm{L}^{-}$ & 3.38 \\
\hline 35 & Calcarine $\mathrm{R}$ to Temporal $\mathrm{Mid} \mathrm{L}$ & 3.81 \\
\hline 36 & Lingual_L to Temporal_Mid_L & 3.65 \\
\hline 37 & Lingual $\mathrm{R}$ to Temporal Mid L & 3.21 \\
\hline 38 & Fusiform_L to Temporal_Mid_L & 3.45 \\
\hline 39 & Precuneus_L to Temporal_Mid_L & 4.18 \\
\hline 40 & Temporal Sup L to Temporal Mid L & 4.35 \\
\hline 41 & Pallidum_R to Temporal_Mid_R & 3.44 \\
\hline 42 & Frontal Inf Tri L to Temporal Pole Mid L & 2.33 \\
\hline 43 & Rolandic Oper L to Temporal Pole Mid L & 2.08 \\
\hline 44 & Hippocampus_L to Temporal_Pole_Mid_L & 3.46 \\
\hline 45 & Temporal_Mid_L to Temporal_Pole_Mid_L & 3.59 \\
\hline 46 & Temporal Sup $\mathrm{R}$ to Temporal Pole ${ }^{-} \mathrm{Mid}^{-} \mathrm{R}$ & 3.25 \\
\hline
\end{tabular}

${ }^{a}$ AAL abbreviations can be found in Table 4.

that survived the FDR correction are presented in Fig 1 and Tables 3 and 4. Connections of the left middle temporal gyrus were the most prevalent hypoconnected connections (10 connections), and among them, the most statistically significantly hypoconnected interlobular connection was the left precuneus-left middle temporal gyrus connection (test statistic: 4.18 ; average FA of 0.34 in the inattention group compared with 0.37 in the group with normal attention), which we explored further. Figure 2 shows an example of the participant's tractography streamlines going through the left precuneus and left middle temporal gyrus. None of the connections were stronger in the inattention group compared with the group with normal attention.
Table 4: AAL abbreviations and standardized names of the corresponding brain regions

\begin{tabular}{|c|c|}
\hline AAL Abbreviation & $\begin{array}{l}\text { Standardized Name of } \\
\text { the Brain Region }\end{array}$ \\
\hline Amygdala & Amygdala \\
\hline Angular & Angular gyrus \\
\hline Calcarine & Calcarine fissure and surrounding cortex \\
\hline Caudate & Caudate nucleus \\
\hline Cingulum_Mid & $\begin{array}{l}\text { Median cingulate and paracingulate gyri } \\
\quad(>20 \% \text { overlap with anterior cingulate })\end{array}$ \\
\hline Cingulum_Post & Posterior cingulate gyrus \\
\hline Frontal_Inf_Oper & Inferior frontal gyrus, opercular part \\
\hline Frontal_Inf_Orb & Inferior frontal gyrus, orbital part \\
\hline Frontal_Inf_Tri & Inferior frontal gyrus, triangular part \\
\hline Frontal_Mid & Middle frontal gyrus \\
\hline Frontal_Mid_Orb & Middle frontal gyrus, orbital part \\
\hline Frontal_Sup_Medial & Superior frontal gyrus, medial \\
\hline Frontal_Sup_Orb & Superior frontal gyrus, orbital part \\
\hline Fusiform & Fusiform gyrus \\
\hline Hippocampus & Hippocampus \\
\hline Lingual & Lingual gyrus \\
\hline Olfactory & Olfactory cortex \\
\hline Pallidum & Lenticular nucleus, pallidum \\
\hline ParaHippocampal & Parahippocampal gyrus \\
\hline Parietal_Inf & $\begin{array}{l}\text { Inferior parietal, excluding supramarginal } \\
\text { and angular gyri }\end{array}$ \\
\hline Parietal_Sup & Superior parietal gyrus \\
\hline Postcentral & Postcentral gyrus \\
\hline Precentral & Precentral gyrus \\
\hline Precuneus & Precuneus \\
\hline Putamen & Lenticular nucleus, putamen \\
\hline Rolandic_Oper & Rolandic operculum \\
\hline Supp_Mötor_Area & Supplementary motor area \\
\hline SupraMarginal & Supramarginal gyrus \\
\hline Temporal_Mid & Middle temporal gyrus \\
\hline Temporal_Pole_Mid & Temporal pole, middle temporal gyrus \\
\hline Temporal_Pole_Sup & Temporal pole, superior temporal gyrus \\
\hline Temporal_Sup & Superior temporal gyrus \\
\hline
\end{tabular}

Neonatal Predictors of Abnormal Structural Connectivity Associated with Attentional Problems in Adolescents

Linear regression analysis was performed with left precuneus-left middle temporal gyrus connectivity as the dependent variable and neonatal clinical and imaging metrics and the age at the adolescent MR imaging visit as independent variables. Only low birth weight in grams and ventriculomegaly detected at the second neonatal scan independently and statistically significantly predicted hypoconnectivity between the left precuneus and left middle temporal gyrus $(t=4.874, P<.000$ and $t=4.617, P<.000$, respectively). None of the other variables showed statistical significance (neonatal clinical characteristics listed in Table 1 or neonatal scan results listed in Table 2, or age at the adolescent MR imaging visit). Similar results were obtained using the average FA as the dependent variable: Low birth weight and ventriculomegaly at the second neonatal scan independently and statistically significantly predicted average FA $(t=2.929, P=.010$ and $t=2.385, P=.031$, respectively). While low birth weight was associated with lower FA, ventriculomegaly was associated with higher FA.

Because only 1 subject had ventriculomegaly, we excluded this subject and performed linear regression with left precuneus-left middle temporal gyrus connectivity as the dependent variable and only birth weight as an independent variable. The results were $t=$ 2.382, $P=.027 ; \mathrm{R}^{2}=0.213$. Figure 3 shows a scatterplot of the 


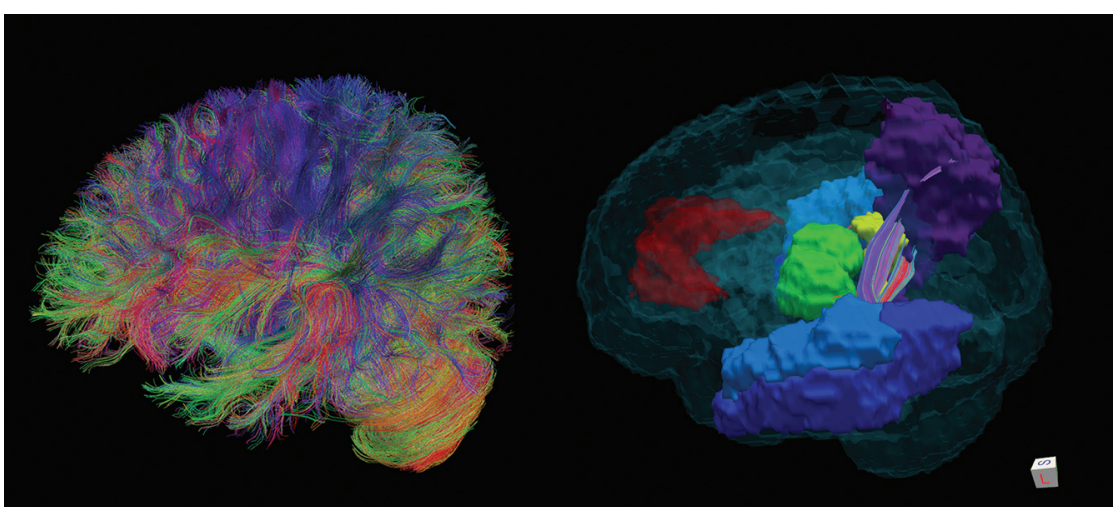

FIG 2. Left: example of study participant's whole-brain tractography. Right: tractography streamlines going through the left precuneus (purple) and left middle temporal gyrus (blue). The anterior cingulate cortex is shown in red; the thalamus, in green.

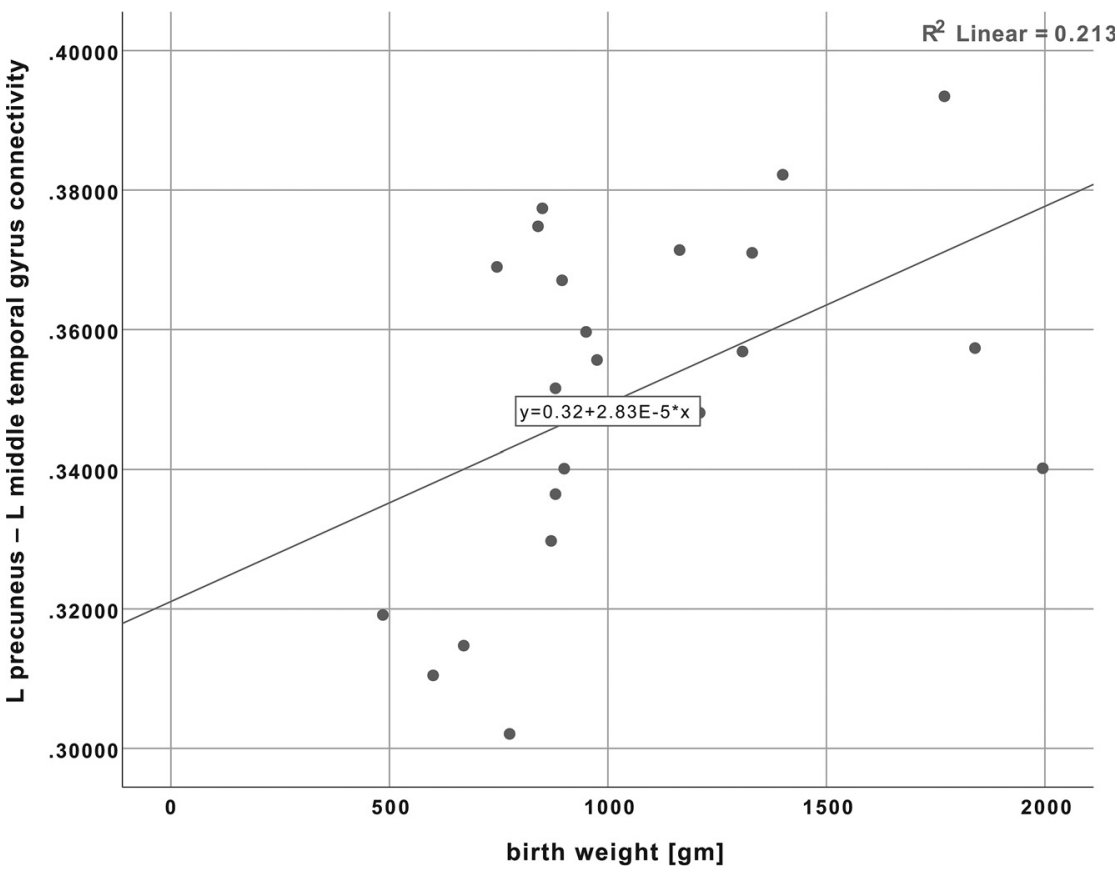

FIG 3. Linear regression: left precuneus-left middle temporal gyrus connectivity as a dependent variable and birth weight as independent variable.

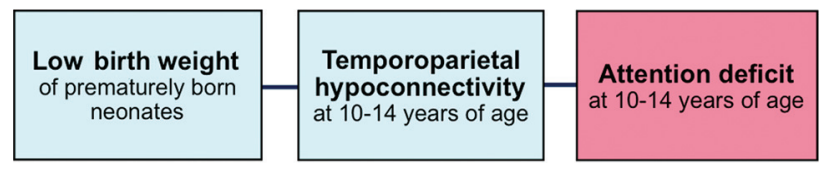

FIG 4. A model that describes the relationship between neonatal characteristics of prematurely born children, structural brain connectivity at adolescence, and attention at adolescence. The model is supported by the findings of this study.

variables. The result remained significant when using birth weight $z$ scores.

\section{DISCUSSION}

The results of this study indicate that attentional problems in adolescents who were born prematurely may be associated with a weaker structural connectivity among the frontal, parietal, temporal, and occipital lobes, including frontal cortices and subcortical regions. The resulting hypoconnected edges linked to atten- tional problems in our study are known to underlie general attentional processes. Specifically, the frontoparietal attentional network is known to facilitate voluntary spatial attention, whereas reorienting of attention recruits other brain areas, such as the temporoparietal junction and lateral inferior frontal cortex. $^{44}$

Our findings are generally supported by previous literature reporting microstructural abnormalities in children formally diagnosed with ADHD and in prematurely born children, as well as by the structural network analysis literature in children with ADHD, ${ }^{18,19}$ though contradictory results have been reported. ${ }^{20}$ Among other regions, we detected lower connectivity of the left middle temporal gyrus to other regions in the subjects with attentional deficits. This finding is aligned with results published by Lei et $\mathrm{al},{ }^{45}$ who observed microstructural abnormalities in the left middle temporal gyrus in children with ADHD, inattentive type. Most interesting, they also observed microstructural disruptions within the right middle temporal gyrus in children with the combined ADHD types compared with controls. Our results indicate a weaker connectivity between the right pallidum and the right middle temporal gyrus in the group with attentional deficits, which is also consistent with those findings.

The conflicting findings within the ADHD literature are, however, of particular interest. Different etiologies might be the reason for some of these differences. Specifically, in a study by Beare et al, ${ }^{20}$ individuals with ADHD presented with a subnetwork of stronger connectivity encompassing bilateral frontostriatal connections as well as left occipital, temporal, and parietal regions, in which the white matter microstructure was associated with ADHD symptom severity. This result differs from those in 2 previous studies, ${ }^{18,19}$ as well as from the study described here. The prematurity status was, however, not reported in these prior studies. The only previous study linking prematurity, ADHD, and white matter metrics is the one by Skranes et al, ${ }^{14}$ which investigated the relationship between clinical findings and FA measurements in white matter of 34 adolescents born prematurely with very low birth weights compared with 47 age-matched controls born at term. In their study, high inattention scores on the ADHD Rating Scale (based on mothers' reports) correlated with low FA values in the right superior and left middle fasciculus.

Previous studies have reported that adolescents with very low birth weights are more likely to have attention and/or executive 
function deficits compared with controls, ${ }^{46,47}$ which is consistent with our findings. In our study, low birth weight and ventriculomegaly (but not white matter injury or intraventricular hemorrhage) detected on the neonatal MR imaging were associated with altered temporoparietal connectivity in adolescence. Because ventriculomegaly was only detected in 1 study participant, we do not draw any conclusions. The obtained results are summarized in the model in Fig 4. A potential explanation for the association of low birth weight (but not gestational age) with temporoparietal hypoconnectivity is the substantial risk of children with very low birth weights for perinatal hemorrhages and injury. ${ }^{48}$ The subsequent hypoconnectivity can be caused by perinatal white matter damage that has long-term effects on myelin disturbances, disorganization, or a reduced number of axons in projectional, commissural, and association tracts. ${ }^{14}$ Although neonatal white matter injury or intraventricular hemorrhage was not associated with hypoconnectivity in adolescence in our study, this may relate to the lower resolution of MR images 14 years ago when some of the neonatal scans were performed, as well as limited power to detect a difference between groups, given the small sample size. Lower birth weight was also associated with other markers of illness severity, which, in turn, were also linked to inattention, and it is likely that unmeasured variables are a source of residual confounding. Additionally, other factors (eg, parenting style, adverse events, and so forth) may have a strong influence on performance in attentional tasks.

Several methodologic limitations need to be considered when interpreting our findings. First, a DTI-based tractography method was used to reconstruct structural brain networks, which is the most widely used tractography method but is limited in terms of its capacity for resolving crossing fibers. ${ }^{49}$ Second, our study did not include a typically developing control group or a group of term-born adolescents with attentional problems. Third, the assessment of attention in creating the binary variable of the attention/inattention group was nonuniform across subjects. Finally, the study sample was limited and included only 24 adolescents who came for the 10- to 14 year follow-up visit and had diffusion MR imaging data. Nevertheless, the main result of an aberrant structural connectivity associated with attentional problems was statistically significant after correction for multiple comparisons. This result contributes to our understanding of the mechanisms underlying the development of attentional deficits in children born prematurely and can help direct future studies.

It would be advantageous if future studies of aberrant neural networks in ADHD included information about the gestational age at birth (prematurity) of study participants. Another important step would be a comparison of 4 groups in 1 study: term-born adolescents without attention deficits, term-born adolescents with attention deficits, pre-term-born adolescents without attention deficits, and pre-term-born adolescents with attention deficits.

\section{CONCLUSIONS}

In this study, we examined network-level neural correlates of attentional deficits in adolescents born prematurely as well as neonatal predictors of those neural correlates and attentional problems. Our major findings are the hypoconnected axonal tracts in the frontal, parietal, temporal, and occipital lobes in adolescents with attentional problems, with temporoparietal connection be- ing the most prominent and low birth weight being a predictor of this hypoconnectivity. This knowledge could lead to a better understanding of the mechanisms that play a role in the development of attentional deficits in children/adolescents born prematurely and could possibly lead to new therapeutic agents and additional interventions.

\section{ACKNOWLEDGMENTS}

We would like to thank all of the study participants and their parents who made this work possible. We also thank Drs Bridget Johnson and Elizabeth Rogers for their help with the psychological assessments of the study participants. Finally, we thank Laurel Haeusslein, Veronica de Santiago, Shrey Goel, and Kristyn Casalino for the study coordination.

Disclosures: Olga Tymofiyeva—RELATED: Grant: National Institutes of Health, Comments: National Institutes of Health grants P01NS082330, R01HD072074, and R21AT009173*; Support for Travel to Meetings for the Study or Other Purposes: National Institutes of Health, Comments: National Institutes of Health grants P01NS082330, R01HD072074, and R21AT009173*. Dawn Gano-RELATED: Grant: National Institutes of Health*; UNRELATED: Grants/Grants Pending: PERF*. Robert J. Trevino-UNRELATED: Other: Summer Research Training Program at the University of California, San Francisco; Maximizing Access to Research Careers-Undergraduate Student Training for Academic Research (MARC U-STAR), Comments: During my time at the University of California, San Francisco, I was in a living arrangement provided by the Summer Research Training Program at the University of California, San Francisco. I am also partly funded by the MARC U-STAR Program through $\mathrm{Na}$ tional Institutes of Health/National Institute of General Medical Sciences MARC U-STAR GM07717. Hannah C. Glass_UNRELATED: medicolegal consulting: various Grants/Grants Pending: National Institutes of Health, Cerebral Palsy Alliance, Pediatric Epilepsy Research Foundation. Donna M. Ferriero-RELATED: Grant: National Institutes of Health*; UNRELATED: Grants/Grants Pending: National Institutes of Health, Cerebral Palsy Alliance*; Royalties: Elsevier for an edited book*. A. James Barkovich—RELATED: Grant: National Institutes of Health, Comments: grant to look at differences in brain injuries and brain structure in children born before 32 weeks' gestational age*. Duan Xu—RELATED: Grant: National Institutes of Health*; UNRELATED: Grants/Grants Pending: National Institutes of Health*. *Money paid to the institution.

\section{REFERENCES}

1. Blencowe H, Cousens S, Oestergaard MZ, et al. National, regional, and worldwide estimates of preterm birth rates in the year 2010 with time trends since 1990 for selected countries: a systematic analysis and implications. Lancet 2012;379:2162-72 CrossRef Medline

2. Aylward GP. Neurodevelopmental outcomes of infants born prematurely. Dev Behav Pediatr 2005;26:427-40 CrossRef

3. Lindström K, Lindblad F, Hjern A. Preterm birth and attentiondeficit/hyperactivity disorder in schoolchildren. Pediatrics 2011; 127:858-65 CrossRef Medline

4. Alexander AL, Lee JE, Lazar M, et al. Diffusion tensor imaging of the brain. Neurotherapeutics 2007;4:316-29 CrossRef Medline

5. Chanraud S, Zahr N, Sullivan EV, et al. MR diffusion tensor imaging: a window into white matter integrity of the working brain. Neuropsychol Rev 2010;20:209-25 CrossRef Medline

6. Silk TJ, Vance A, Rinehart N, et al. White-matter abnormalities in attention deficit hyperactivity disorder: a diffusion tensor imaging study. Hum Brain Mapp 2009;30:2757-65 CrossRef Medline

7. Peterson DJ, Ryan M, Rimrodt SL, et al. Increased regional fractional anisotropy in highly screened attention-deficit hyperactivity disorder (ADHD). J Child Neurol 2011;26:1296-302 CrossRef Medline

8. Tamm L, Barnea-Goraly N, Reiss AL. Diffusion tensor imaging reveals white matter abnormalities in attention-deficit/hyperactivity disorder. Psychiatry Res 2012;202:150-54 CrossRef Medline

9. Ashtari M, Kumra S, Bhaskar SL, et al. Attention-deficit/hyperactivity disorder: a preliminary diffusion tensor imaging study. Biol Psychiatry 2005;57:448-55 CrossRef Medline

10. Makris N, Buka SL, Biederman J, et al. Attention and executive systems 
abnormalities in adults with childhood ADHD: a DT-MRI study of connections. Cereb Cortex 2008;18:1210-20 CrossRef Medline

11. Bouziane C, Caan MW, Tamminga HG, et al. ADHD and maturation of brain white matter: A DTI study in medication naive children and adults. Neuroimage Clin 2018;17:53-59 CrossRef Medline

12. Nagy Z, Westerberg H, Skare S, et al. Preterm children have disturbances of white matter at 11 years of age as shown by diffusion tensor imaging. Pediatr Res 2003;54:672-79 CrossRef Medline

13. Vangberg TR, Skranes J, Dale AM, et al. Changes in white matter diffusion anisotropy in adolescents born prematurely. Neuroimage 2006;32:1538-48 CrossRef Medline

14. Skranes J, Vangberg TR, Kulseng S, et al. Clinical findings and white matter abnormalities seen on diffusion tensor imaging in adolescents with very low birth weight. Brain 2007;130(Pt 3):654-66 CrossRef Medline

15. Allin MP, Kontis D, Walshe M, et al. White matter and cognition in adults who were born preterm. PLoS One 2011;6:e24525 CrossRef Medline

16. Mullen KM, Vohr BR, Katz KH, et al. Preterm birth results in alterations in neural connectivity at age $\mathbf{1 6}$ years. Neuroimage 2011;54: 2563-70 CrossRef Medline

17. Meskaldji DE, Fischi-Gomez E, Griffa A, et al. Comparing connectomes across subjects and populations at different scales. Neuroimage 2013;80:416-25 CrossRef Medline

18. Cao Q, Shu N, An L, et al. Probabilistic diffusion tractography and graph theory analysis reveal abnormal white matter structural connectivity networks in drug-naive boys with attention deficit/hyperactivity disorder. J Neurosci 2013;33:10676-87 CrossRef Medline

19. Hong SB, Zalesky A, Fornito A, et al. Connectomic disturbances in attention-deficit/hyperactivity disorder: a whole-brain tractography analysis. Biol Psychiatry 2014;76:656-63 CrossRef Medline

20. Beare R, Adamson C, Bellgrove MA, et al. Altered structural connectivity in ADHD: a network-based analysis. Brain Imaging Behav 2017;11:846-58 CrossRef Medline

21. Bethlehem RA, Romero-Garcia R, Mak E, et al. Structural covariance networks in children with autism or ADHD. Cereb Cortex 2017;27: 4267-76 CrossRef Medline

22. Rouse DJ, Landon M, Leveno KJ, et al; National Institute of Child Health and Human Development, Maternal-Fetal Medicine Units Network. The Maternal-Fetal Medicine Units cesarean registry: chorioamnionitis at term and its duration-relationship to outcomes. Am J Obstet Gynecol 2004;191:211-16 CrossRef Medline

23. Kliegman RM, Hack M, Jones $P$, et al. Epidemiologic study of necrotizing enterocolitis among low-birth-weight infants: absence of identifiable risk factors. J Pediatr 1982;100:4404 Medline

24. Dumoulin CL, Rohling KW, Piel JE, et al. Magnetic resonance imaging compatible neonate incubator. Concepts Magn Reson 2002;15: 117-28 CrossRef

25. Miller SP, Cozzio CC, Goldstein RB, et al. Comparing the diagnosis of white matter injury in premature newborns with serial MR imaging and transfontanel ultrasonography findings. AJNR Am J Neuroradiol 2003;24:1661-69 Medline

26. Miller SP, Ferriero DM, Leonard C, et al. Early brain injury in premature newborns detected with magnetic resonance imaging is associated with adverse early neurodevelopmental outcome. J Pediatr 2005;147:609-16 CrossRef Medline

27. Papile LA, Burstein J, Burstein R, et al. Incidence and evolution of subependymal and intraventricular hemorrhage: a study of infants with birth weights less than 1,500 gm. J Pediatr 1978;92:529-34 CrossRef Medline

28. Greenberg LM. Test of Variables of Attention. Los Alamitos: The Tova Company; 1991

29. Achenbach TM, Rescorla LA. Manual for the ASEBA School-Age Forms and Profiles: An Integrated System of Multi-Informant Assessment. Burlington: ASEBA; 2001

30. Wu YY, Huang YS, Chen YY, et al. Psychometric study of the test of variables of attention: preliminary findings on Taiwanese children with attention-deficit/hyperactivity disorder. Psychiatry Clin Neurosci 2007;61:211-18 CrossRef Medline

31. Chang LY, Wang MY, Tsai PS. Diagnostic accuracy of rating scales for attention-deficit/hyperactivity disorder: a meta-analysis. Pediatrics 2016;137:e20152749 CrossRef Medline

32. Morales-Hidalgo P, Hernández-Martínez C, Vera M, et al. Properties of the Conners-3 and Conners Early Childhood Indexes in a Spanish school population. International Journal of Clinical and Health Psychology 2017;17:85-96 CrossRef

33. Forbes GB. Clinical utility of the Test of Variables of Attention (TOVA) in the diagnosis of attention-deficit/hyperactivity disorder. J Clin Psychol 1998;54:461-76 CrossRef Medline

34. Smith SM, Jenkinson M, Woolrich MW, et al. Advances in functional and structural MR image analysis and implementation as FSL. Neuroimage 2004;23(Suppl 1):S208-19 CrossRef Medline

35. Tymofiyeva O, Hess CP, Ziv E, et al. Towards the "baby connectome": mapping the structural connectivity of the newborn brain. PLoS One 2012;7:e31029 CrossRef Medline

36. Wang R, Benner T, Sorensen AG, et al. Diffusion Toolkit: a software package for diffusion imaging data processing and tractography. In: Proceedings of Annual Meeting of the International Society for Magnetic Resonance in Medicine and the European Society for Magnetic Resonance in Medicine and Biology and Society for MR Radiographers e Technologists, Berlin, Germany. May 19-25, 2007:3720

37. Mori S, Crain BJ, Chacko VP, et al. Three-dimensional tracking of axonal projections in the brain by magnetic resonance imaging. Ann Neurol 1999;45:265-69 CrossRef Medline

38. Tymofiyeva O, Connolly CG, Ho TC, et al. DTI-based connectome analysis of adolescents with major depressive disorder reveals hypoconnectivity of the right caudate. J Affect Disord 2017;207:18-25 CrossRef Medline

39. Tzourio-Mazoyer N, Landeau B, Papathanassiou D, et al. Automated anatomical labeling of activations in SPM using a macroscopic anatomical parcellation of the MNI MRI single-subject brain. Neuroimage 2002;15:273-89 CrossRef Medline

40. Jenkinson M, Smith S. A global optimisation method for robus affine registration of brain images. Med Image Anal 2001;5:143-56 CrossRef Medline

41. Jenkinson M, Bannister P, Brady M, et al. Improved optimization for the robust and accurate linear registration and motion correction of brain images. Neuroimage 2002;17:825-41 CrossRef Medline

42. Zalesky A, Fornito A, Bullmore ET. Network-based statistic: identifying differences in brain networks. Neuroimage 2010;53:1197-207 CrossRef Medline

43. Nass RD. Evaluation and assessment issues in the diagnosis of attention deficit hyperactivity disorder. Semin Pediatr Neurol 2005; 12: 200-16 CrossRef Medline

44. Gazzaniga MS, Ivry RB, Mangun GR. Cognitive Neuroscience: The Biology of the Mind. 3rd ed. Rockville: W.W. Norton \& Company; 2008:553

45. Lei D, Ma J, Du X, et al. Microstructural abnormalities in the combined and inattentive subtypes of attention deficit hyperactivity disorder: a diffusion tensor imaging study. Sci Rep 2014;4:6875 CrossRef Medline

46. Indredavik MS, Vik T, Heyerdahl S, et al. Psychiatric symptoms in low birth weight adolescents, assessed by screening questionnaires. Eur Child Adolesc Psychiatry 2005;14:226-36 CrossRef Medline

47. Kulseng S, Jennekens-Schinkel A, Naess P, et al. Very-low-birthweight and term small-for-gestational-age adolescents: attention revisited. Acta Paediatr 2006;95:224-30 CrossRef Medline

48. Volpe JJ. Brain injury in the premature infant: neuropathology, clinical aspects, pathogenesis, and prevention. Clin Perinatol 1997; 24:567-87 CrossRef Medline

49. Farquharson S, Tournier JD, Calamante F, et al. White matter fiber tractography: why we need to move beyond DTI. J Neurosurg 2013; 118:1367-77 CrossRef Medline 